in our understanding of how nascent endocytic vesicles are disengaged from the machinery that produced them. Future studies promise to reveal further details of how actin polymerization and endocytic vesicle formation are spatially and temporally coordinated in yeast and mammalian cells.

1. Merrifield, C.J. Trends Cell Biol. 14, 352-358 (2004).

2. Engqvist-Goldstein, A. E. \& Drubin, D. G. Annu. Rev.
Cell Dev. Biol. 19, 287-332 (2003).

3. Yarar, D., Waterman-Storer, C. M. \& Schmid, S. L. Mol. Biol. Cell 16, 964-975 (2005).

4. Lemmon, S. K. Curr. Biol. 11, R49-52 (2001).

5. Toshima, J., Toshima, J. Y., Martin, A. C. \& Drubin, D. G. Nature Cell Biol. 7, 246-254 (2005).

6. Weaver, A. M., Young, M.E., Lee, W. L. \& Cooper, J. A Curr. Opin. Cell Biol. 15, 23-30 (2003).

7. Kaksonen, M., Sun, Y. \& Drubin, D. G. Cel/ 115, 475487 (2003).

8. Smythe, E. \& Ayscough, K. R. EMBO Rep. 4, 246-251 (2003).

9. Sekiya-Kawasaki, M. et al. J. Cell Biol. 162, 765-772 (2003).
10. Miliaras, N. B., Park, J.H. \& Wendland, B. Traffic 5, 963-978 (2004)

11. Hertzog, M. et al. Cell 117, 611-623 (2004).

12. Duncan, M.C., Cope, M.J., Goode, B.L., Wendland, B. \& Drubin, D.G. Nature Cell Biol. 3, 687-690 (2001).

13. Huang, B., Zeng, G., Ng, A. Y. \& Cai, M. Mol. Biol. Cell 14, 4871-4884 (2003).

14. Henry, K.R. et al. Curr. Biol. 13, 1564-1569 (2003).

15. Zhang, L., Gjoerup, O. \& Roberts, T. M. Proc. Natl Acad. Sci. USA 101, 10296-10301 (2004).

\section{Growth factor control of autophagy}

Autophagy, the process whereby cells begin to self digest, has long been suspected to aid organismal survival in times of trouble. Reporting in the 28 January issue of Cell, Thompson and colleagues (Cell, doi: 10.1016/ j.cell.2004.11.046; 2005) now show that removal of growth factor can trigger autophagy in haematopoietic cells and that this prolongs cell survival.

So far, we know of three processes that can influence whether a cell chooses to live or die: programmed cell death, or apoptosis; necrosis, a death mechanism that also triggers inflammation and an immune response; and autophagy. This last process is induced when nutrient and/or growth factors are lacking. During autophagy, parts of the cytoplasm and organelles are encapsulated by intracellular vesicles called autophagosomes, which then fuse with lysosomes and are degraded. In this way, autophagy helps to provide an intracellular source of energy. Intuitively, you would imagine that this would help cells to survive, but there have been mixed reports as to whether the ultimate outcome is one of survival or death.

Because the stimuli for apoptosis and autophagy often overlap, it has been difficult to identify the crucial factors that induce autophagy. To get around this problem Lum et al. generated a haematopoietic cell line, dependent on the growth factor IL-3, that also lacks the essential apoptotic regulators Bax and Bak, so leaving the cells immune to apoptosis. This allowed the researchers to investigate the effect of extracellular growth factor and nutrient withdrawal on autophagy and cell survival.

They found that removal of IL-3 alone is sufficient to trigger autophagy: the cells formed autophagosomes, lost cytoplasmic structures and began to use intracellular resources to maintain their metabolism. Remarkably, without IL-3, cells continued to use only intracellular energy sources even when extracellular nutrients were available. Only re-addition of IL-3 rescued the cells' ability to take up extracellular nutrients.

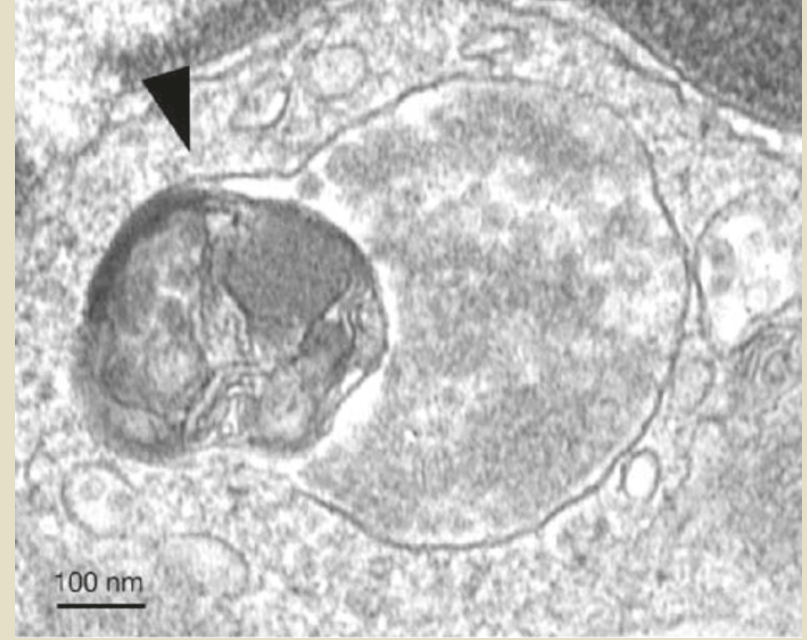

Late autophagosome (indicated by arrow) fusing with a lysosome. Figure reproduced with permission from Cell, doi: 10.1016/j.cell.2004.11.046 ๑ (2005) with permission from Elsevier.

Ultimately, the cells lacking growth factor did die, posing the question of whether autophagy is promoting or preventing cell death. To address this, the authors asked what would happen if they inhibited autophagy; the outcome was a rapid decrease in ATP production and cell death. So, the study clearly shows that autophagy is a survival mechanism that prolongs the life of cells that are deprived of growth factors, at least for mammalian haematopoietic cells. Interestingly, it also highlights that cells can eventually undergo autonomous cell death in the absence of apoptosis, simply by reducing the availability of extracellular growth factors such as IL-3. The mechanisms driving this alternative death pathway remain to be seen.

MARKUS WAGNER 